\title{
On asymptotic behavior of fractional Cauchy transform
}

\author{
Igor Chyzhykov ${ }^{1,2} \cdot$ Galyna Beregova $^{3}$
}

Received: 8 October 2018 / Accepted: 24 February 2019 / Published online: 6 June 2019

(c) The Author(s) 2019

\section{Abstract}

We study non-regularity of growth of the fractional Cauchy transform

$$
f(z)=\int_{-\pi}^{\pi} \frac{d \psi(t)}{\left(1-z e^{-i t}\right)^{\alpha}}, \quad \alpha>0, \psi \in B V[-\pi, \pi],
$$

in terms of the modulus of continuity of the function $\psi$. Sharp estimates of the lower logarithmic order of $f$ are found. In the case $\alpha \in(0,1)$ the estimates are of different form than that for the logarithmic order.

Keywords Fractional Cauchy transform · Modulus of continuity · Lower logarithmic order $\cdot$ Lipschitz class · Maximum modulus

\section{Introduction}

Let $\mathbb{D}=\{z \in \mathbb{C}:|z|<1\}$ denote the unit disc, $\alpha>0 ;(1-w)^{\alpha}, w \in \mathbb{D}$, be the principle branch of the power function, i.e. $\left.(1-w)^{\alpha}\right|_{w=0}=1$.

Our main focus is to study of the following Cauchy type integral

$$
f(z)=\int_{-\pi}^{\pi} \frac{d \psi(t)}{\left(1-z e^{-i t}\right)^{\alpha}},
$$

Igor Chyzhykov

chyzhykov@matman.uwm.edu.pl

Galyna Beregova

gberegova@yahoo.com

1 School of Mathematics Science, Guizhou Normal University, No. 116 Baoshan Road (N), Guiyang 550001, Guizhou, China

2 Present Address: Faculty of Mathematics and Computer Science, Warmia and Mazury University of Olsztyn, Soneczna 54, 10710 Olsztyn, Poland

3 Department of Computational Mathematics and Programming, Institute of Applied Mathematics and Fundamental Sciences, National University Lviv Polytechnic, 5 Mytropolyt Andrei str., Buiding 4, Lviv, Ukraine 
where $\psi$ is a complex-valued function of bounded variation, i.e., $\psi \in B V[-\pi, \pi]$, or a fractional Cauchy transform of the measure $\psi^{*}$ on $\partial \mathbb{D}$ associated with $\psi$. The family of all functions of the form (1) is denoted by $\mathcal{F}_{\alpha}$. A study of $\mathcal{F}_{\alpha}$ can be found in the book [11], here we mention only few properties. $\mathcal{F}_{\alpha}$ is a Banach space with respect to the norm $\|f\|_{\mathcal{F}_{\alpha}}=\inf _{\psi^{*}}\left\|\psi^{*}\right\|$, where $\left\|\psi^{*}\right\|$ denotes the total variation of $\psi^{*}$. A straightforward consequence of the definition is that for $f \in \mathcal{F}_{\alpha}$ one has

$$
M(r, f) \leq \frac{\left\|\psi^{*}\right\|}{(1-r)^{\alpha}}
$$

where $M(r, f)=\max \left\{\left|f\left(r e^{i \theta}\right)\right|:|z|=r\right\}$.

Many relations with Hardy, Besov, and Dirichlet-type spaces are obtained in the mentioned monograph.

Theorem A If $0<\alpha \leq 1$, then $\mathcal{F}_{\alpha} \subset H^{p}$ for $0<p<1 / \alpha$. If $0<p \leq 1$ then $H^{p} \subset \mathcal{F}_{1 / p}$, where $H^{p}$ is the Hardy space of analytic functions in $\mathbb{D}$ satisfying

$$
\sup _{0<r<1} \int_{-\pi}^{\pi}\left|f\left(r e^{i \theta}\right)\right|^{p} d \theta<\infty
$$

Theorem B If $0<\alpha<\beta$, then $\mathcal{B}_{\alpha} \subset \mathcal{F}_{\alpha} \subset \mathcal{B}_{\beta}$, where $\mathcal{B}_{\alpha}$ is the Besov space of analytic functions in $\mathbb{D}$ satisfying

$$
\int_{\mathbb{D}}\left|f^{\prime}(z)\right|(1-|z|)^{\alpha-1} d m(z)<\infty
$$

where $m$ is the planar Lebesgue measure.

Functions from $\mathcal{F}_{\alpha}$ appear frequently in representation theorems for different classes of functions defined on the unit disc $\mathbb{D}$. In particular, Djrbashian introduced [5] classes $A_{\alpha}, \alpha>-1$, of analytic functions in $\mathbb{D}$. For $\alpha>0$, an analytic function $f$ belongs to the class $A_{\alpha}, \alpha>0$, if and only if

$$
\sup _{0<r<1} \int_{0}^{2 \pi}\left(\int_{0}^{r}(r-t)^{\alpha-1} \log \left|f\left(t e^{i \varphi}\right)\right| d t\right)^{+} d \varphi<+\infty .
$$

Note that the union of $\bigcup_{\alpha>0} A_{\alpha}$ contains the class of analytic functions $f$ in $\mathbb{D}$ of finite order of growth, that is $\log \log M(r, f)=O\left(\log \frac{1}{1-r}\right)(r \rightarrow 1-)$. Due to results of Djrbashian [5, Chap. IX] functions $f \in \mathcal{F}_{\alpha+1}$ appear in a parametric representation of the class $A_{\alpha}, \alpha>-1$.

Radial and non-tangential limits of $f \in \mathcal{F}_{\alpha}$ were investigated in many papers, e.g., Hallenbeck and MacGregor [8,9], and Sheremeta [13], see also [11, Chap. 10; 12]. It turns out that the estimate (2) can be improved in terms of the modulus of continuity for $\psi$. Let 


$$
\omega(\delta, \psi)=\sup \{|\psi(x)-\psi(y)|:|x-y|<\delta, x, y \in[-\pi, \pi]\}
$$

be the modulus of continuity of $\psi$.

Denote by $\Lambda_{\gamma}$ [15] the class of functions $\psi$ such that $\omega(\delta, \psi)=O\left(\delta^{\gamma}\right), \gamma \in(0,1]$. It comes from Hardy and Littlewood [10], see also [6,14], that there is a strong interplay between smoothness of a measure (or associated function $\psi$ of bounded variation) and the growth of integral (1).

The next result in this spirit is a consequence of Theorem 3.4 [4] (see also Theorem $3.1[1])$.

Theorem C Let $f$ be an analytic function in $\mathbb{D}$ of the form (1). Let $\alpha>\gamma$ and $0<$ $\gamma \leq 1$. Then $\psi \in \Lambda_{\gamma}$, if and only if

$$
M(r, f)=O\left((1-r)^{\gamma-\alpha}\right), \quad r \rightarrow 1-.
$$

Remark 1 More general results using the concept of a proximate order are obtained in [4]. There are counterparts for harmonic functions represented by Poisson-type integrals as well, see $[1,3]$.

Remark 2 Growth of $p$ th means of functions $f \in \mathcal{F}_{\alpha}$ is described in [2].

A common feature of the above mentioned results is that the growth of $f$ depends on which Hölder class $\Lambda_{\gamma}$ the modulus of continuity of $\psi$ belongs to. Let

$$
p[f]:=\limsup _{r \rightarrow 1-} \frac{\log M(r, f)}{\log \frac{1}{1-r}}, \quad \lambda[f]:=\liminf _{r \rightarrow 1-} \frac{\log M(r, f)}{\log \frac{1}{1-r}}
$$

denote the logarithmic order and the lower logarithmic order, respectively. It follows from Theorem $\mathrm{C}$ that for an arbitrary $f \in \mathcal{F}_{\alpha}$ and appropriate $\alpha, \gamma$ we have $p[f] \leq$ $\alpha-\gamma$ if and only if $\psi \in \Lambda_{\gamma}$. The following question appears naturally. Does a smoothness of the modulus of continuity $\omega(\delta, \psi)$ on a sequence $\left(\delta_{n}\right)$ of values $\delta$ tending to zero imply an upper estimate for the lower logarithmic order similar to that for the logarithmic order?

We shall see that the answer is in the positive provided that $\alpha>1$, but in general, the situation is more complicated.

Theorem 3 Let $\alpha>0,0 \leq \gamma<\lambda<1$.

Suppose that $\omega\left(\delta_{n}, \psi\right)=O\left(\delta_{n}^{\lambda}\right), \delta_{n} \rightarrow 0+$, while $\omega(\delta, \psi)=O\left(\delta^{\gamma}\right), \delta \rightarrow 0+$, and that $f$ is of the form (1).

(i) If $0<\alpha<\gamma$, then $M(r, f)=O(1)$.

(ii) If $0<\alpha=\gamma$, then $M(r, f)=O\left(\log \frac{1}{1-r}\right)$.

(iii) If $\gamma<\alpha$, then

$$
M\left(r_{n}, f_{\alpha}\right)=O\left(\left(\frac{1}{1-r_{n}}\right)^{\Delta}\right), \quad n \rightarrow \infty
$$


on a sequence $r_{n} \rightarrow 1-(n \rightarrow \infty)$, where

$$
\Delta= \begin{cases}\frac{\alpha(\alpha-\gamma)(1-\lambda)}{\alpha(1-\lambda)+\lambda-\gamma}, & \alpha<1 \\ \alpha-\lambda, & \alpha>1\end{cases}
$$

and, for $\alpha=1$

$$
M\left(r_{n}, f_{\alpha}\right)=O\left(\left(\frac{1}{1-r_{n}}\right)^{1-\varkappa_{2}} \log \frac{1}{1-r_{n}}\right), \quad n \rightarrow \infty
$$

Remark 4 The quantity $\Delta$ in (4) is continuous at 1 as a function of $\alpha$. Moreover, $\Delta \rightarrow \alpha-\lambda$ as $\gamma \rightarrow \lambda$.

Remark 5 Any modulus of continuity $\omega$ satisfies [7, Chap. 3]

$$
\frac{\omega\left(t_{2}\right)}{t_{2}} \leq \frac{2 \omega\left(t_{1}\right)}{t_{1}}, \quad 0<t_{1}<t_{2}
$$

If $\omega\left(\delta_{n}, \psi\right) \leq C \delta_{n}, \delta_{n} \rightarrow 0+$, i.e., $\lambda=1$, then

$$
\omega(\delta, \psi) \leq 2 C \delta, \quad \delta>\delta_{n}
$$

so $\psi \in \Lambda_{1}$, and $\gamma=\lambda=1$. This is why we exclude this case in the assumptions of Theorem 3.

Corollary 1 Let $\alpha>0$. Suppose that for arbitrary $\varepsilon>0$ there exists a sequence $\delta_{n} \rightarrow 0+(n \rightarrow \infty)$ such that $\omega\left(\delta_{n}, \psi\right)=O\left(\delta_{n}^{\lambda-\varepsilon}\right)(n \rightarrow \infty)$, while $\psi \in \Lambda_{\gamma}$, $0 \leq \gamma<\lambda<1, \gamma<\alpha$. Then

$$
\lambda[f] \leq \begin{cases}\frac{\alpha(\alpha-\gamma)(1-\lambda)}{\alpha(1-\lambda)+\lambda-\gamma}, & \alpha<1 \\ \alpha-\lambda, & \alpha \geq 1\end{cases}
$$

Proof of Theorem 3 Let $\varphi$ be arbitrary on $[-\pi, \pi]$. We extend $\psi$ on $\mathbb{R}$ by the formula $\psi(t+2 \pi)-\psi(t)=\psi(\pi)-\psi(-\pi)$. Since the kernel of the integral (1) is a $2 \pi-$ periodic function in $t$, integrating by parts gives us

$$
\begin{aligned}
f_{\alpha}\left(r e^{i \varphi}\right) & =\int_{-\pi+\varphi}^{\pi+\varphi} \frac{d(\psi(t)-\psi(\varphi))}{\left(1-r e^{i(\varphi-t)}\right)^{\alpha}} \\
& =\frac{\psi(\pi)-\psi(-\pi)}{(1+r)^{\alpha}}+i r \alpha \int_{-\pi}^{\pi} \frac{e^{-i \tau}(\psi(\tau+\varphi)-\psi(\varphi)) d \tau}{\left(1-r e^{-i \tau}\right)^{\alpha+1}} .
\end{aligned}
$$

Therefore, uniformly in $\varphi, \omega(\delta):=\omega(\delta ; \psi)$

$$
\left|f_{\alpha}\left(r e^{i \varphi}\right)\right| \leq 2 \alpha \int_{0}^{\pi} \frac{\omega(x) d x}{\left|1-r e^{i x}\right|^{\alpha+1}}+O(1) \leq 2 \alpha \int_{0}^{1} \frac{\omega(x) d x}{\left|1-r e^{i x}\right|^{\alpha+1}}+O(1) .
$$


We start with the simple cases (i) and (ii). Suppose that $0<\alpha \leq \gamma$. Then $\omega(\delta)=$ $O\left(\delta^{\gamma}\right)$, and standard estimates yield

$$
\int_{0}^{1} \frac{\omega(x) d x}{\left|1-r e^{i x}\right|^{\alpha+1}} \leq \int_{0}^{1-r} \frac{C x^{\gamma} d x}{(1-r)^{\alpha+1}}+\int_{1-r}^{1} \frac{d x}{C x^{\alpha+1-\gamma}}
$$

where $C$ is a positive constant. Hence, the statements (i) and (ii) follow.

Let now $\alpha>\gamma \geq 0$. Consider two cases. First, we suppose that $\alpha<1$. We then choose

$$
\tilde{\delta}_{n}:=\delta_{n}^{\frac{\alpha(1-\lambda)+\lambda-\gamma}{\alpha(1-\gamma)}}=\delta_{n}^{1+\frac{(\lambda-\gamma)(1-\alpha)}{1-\gamma}} .
$$

Note that $\tilde{\delta}_{n} \leq \delta_{n}$, because $\alpha \leq 1$.

In what follows the inequality $g_{1}(n) \lesssim g_{2}(n)$ means that there exists a constant $C$ independent of $n$ such that $g_{1}(n) \leq C g_{2}(n)(n \rightarrow \infty)$.

We put $r_{n}=1-\tilde{\delta}_{n}$. Since a modulus of continuity is nondecreasing, we have

$$
\int_{0}^{\tilde{\delta}_{n}} \frac{\omega(x) d x}{\left|1-r_{n} e^{i x}\right|^{\alpha+1}} \leq \omega\left(\delta_{n}\right) \int_{0}^{\tilde{\delta}_{n}} \frac{d x}{\left(1-r_{n}\right)^{\alpha+1}} \lesssim \frac{\delta_{n}^{\lambda}}{\tilde{\delta}_{n}^{\alpha}} .
$$

Then

$$
\int_{\tilde{\delta}_{n}}^{\delta_{n}} \frac{\omega(x) d x}{\left|1-r_{n} e^{i x}\right|^{\alpha+1}} \lesssim \delta_{n}^{\lambda} \int_{\tilde{\delta}_{n}}^{\delta_{n}} \frac{d x}{x^{\alpha+1}} \lesssim \frac{\delta_{n}^{\lambda}}{\tilde{\delta}_{n}^{\alpha}}
$$

Taking into account (5), and setting $\hat{\delta}_{n}=\delta_{n}^{\frac{1-\lambda}{1-\gamma}}$, we obtain

$$
\int_{\delta_{n}}^{\hat{\delta}_{n}} \frac{\omega(x) d x}{\left|1-r_{n} e^{i x}\right|^{\alpha+1}} \lesssim \int_{\delta_{n}}^{\hat{\delta}_{n}} \frac{\delta_{n}^{\lambda} x d x}{\delta_{n} x^{\alpha+1}} \lesssim \frac{\hat{\delta}_{n}^{1-\alpha}}{\delta_{n}^{1-\lambda}}=\frac{1}{\delta_{n}^{\frac{(1-\lambda)(\alpha-\gamma)}{1-\gamma}}}
$$

Similarly

$$
\int_{\hat{\delta}_{n}}^{1} \frac{\omega(x) d x}{\left|1-r_{n} e^{i x}\right|^{\alpha+1}} \lesssim \int_{\hat{\delta}_{n}}^{1} \frac{x^{\gamma} d x}{x^{\alpha+1}} \lesssim \frac{1}{\hat{\delta}_{n}^{\alpha-\gamma}}=\frac{1}{\delta_{n}^{\frac{(1-\lambda)(\alpha-\gamma)}{1-\gamma}}}
$$

Finally, taking into account that $\tilde{\delta}_{n} \lesssim \delta_{n}$, from the above estimates we deduce

$$
\begin{aligned}
\int_{0}^{1} \frac{\omega(x) d x}{\left|1-r_{n} e^{i x}\right|^{\alpha+1}} & \lesssim \frac{\delta_{n}^{\lambda}}{\tilde{\delta}_{n}^{\alpha}}+\frac{1}{\delta_{n}^{\frac{(1-\lambda)(\alpha-\gamma)}{1-\gamma}}} \leq \frac{\tilde{\delta}_{n}^{\frac{\lambda \alpha(1-\gamma)}{\alpha(1-\lambda)+\lambda-\gamma}}}{\tilde{\delta}_{n}^{\alpha}}+\frac{1}{\tilde{\delta}_{n}^{\frac{\alpha(1-\gamma)}{\alpha(1-\lambda)+\lambda-\gamma}} \frac{(1-\lambda)(\alpha-\gamma)}{1-\gamma}} \\
& \leq \frac{1}{\tilde{\delta}_{n}^{\frac{\alpha(\alpha-\gamma)(1-\lambda)}{\alpha(1-\lambda)+\lambda-\gamma}}}+\frac{1}{\tilde{\delta}_{n}^{\frac{\alpha(\alpha-\gamma)(1-\lambda)}{\alpha(1-\lambda)+\lambda-\gamma}}} \lesssim \frac{1}{\tilde{\delta}_{n}^{\frac{\alpha(\alpha-\gamma)(1-\lambda)}{\alpha(1-\lambda)+\lambda-\gamma}}}
\end{aligned}
$$


This finishes the proof in the first case.

Let now $\alpha>1$. We put $r_{n}:=1-\delta_{n}$ in this case. Arguing similarly as in the first case, we obtain

$$
\int_{0}^{\delta_{n}} \frac{\omega(x) d x}{\left|1-r_{n} e^{i x}\right|^{\alpha+1}} \lesssim \delta_{n}^{\lambda} \int_{0}^{\delta_{n}} \frac{d x}{\left(1-r_{n}\right)^{\alpha+1}} \lesssim \frac{1}{\delta_{n}^{\alpha-\lambda}}
$$

Then

$$
\int_{\delta_{n}}^{\hat{\delta}_{n}} \frac{\omega(x) d x}{\left|1-r_{n} e^{i x}\right|^{\alpha+1}} \lesssim \int_{\delta_{n}}^{\hat{\delta}_{n}} \frac{\delta_{n}^{\lambda} x d x}{\delta_{n} x^{\alpha+1}} \lesssim \frac{1}{\delta_{n}^{\alpha-\lambda}}
$$

Similarly, as in the first case,

$$
\int_{\hat{\delta}_{n}}^{1} \frac{\omega(x) d x}{\left|1-r_{n} e^{i x}\right|^{\alpha+1}} \lesssim \int_{\hat{\delta}_{n}}^{1} \frac{x^{\gamma} d x}{x^{\alpha+1}} \lesssim \frac{1}{\hat{\delta}_{n}^{\alpha-\gamma}}
$$

Thus, using the definition of $\hat{\delta}_{n}$ and the latter estimates we deduce

$$
\int_{0}^{1} \frac{\omega(x) d x}{\left|1-r_{n} e^{i x}\right|^{\alpha+1}} \lesssim \frac{1}{\delta_{n}^{\alpha-\lambda}}+\frac{1}{\hat{\delta}_{n}^{\alpha-\gamma}}=\frac{1}{\delta_{n}^{\alpha-\lambda}}+\frac{1}{\delta_{n}^{\frac{(1-\lambda)(\alpha-\gamma)}{1-\gamma}}} \lesssim \frac{1}{\delta_{n}^{\alpha-\lambda}},
$$

because the inequality $\frac{(1-\lambda)(\alpha-\gamma)}{1-\gamma}<\alpha-\lambda$ is equivalent to $\alpha>1$ provided that $\alpha>\gamma$ and $\gamma<1$.

In the case $\alpha=1$, all the estimates for the case $\alpha>1$ except (14) remain true. Instead of (14) we have

$$
\int_{\delta_{n}}^{\hat{\delta}_{n}} \frac{\omega(x) d x}{\left|1-r_{n} e^{i x}\right|^{2}} \lesssim \int_{\delta_{n}}^{\hat{\delta}_{n}} \frac{\delta_{n}^{\lambda} x d x}{\delta_{n} x^{2}} \lesssim \frac{1}{\delta_{n}^{1-\lambda}} \log \frac{1}{\delta_{n}}
$$

The theorem is proved.

\section{Example of a function of non-regular growth}

The following theorem shows the sharpness of the corollary.

Theorem 6 Let $0<\varkappa_{1}<\varkappa_{2}<1, \varkappa_{1}<\alpha$, and suppose that $\alpha$ is not an odd number. There exists an analytic function $f_{\alpha}$ in $\mathbb{D}$ of the form (1) such that $\omega\left(\delta_{n}, \psi\right)=O\left(\delta_{n}^{\varkappa 2}\right)$, $\delta_{n} \rightarrow 0+$, while $\psi \in \Lambda_{\varkappa_{1}}, p\left[f_{\alpha}\right]=\alpha-\varkappa_{1}$, and

$$
\lambda\left[f_{\alpha}\right]= \begin{cases}\frac{\alpha\left(\alpha-\varkappa_{1}\right)\left(1-\varkappa_{2}\right)}{\alpha\left(1-\varkappa_{2}\right)+\varkappa_{2}-\varkappa_{1}}, & \alpha<1 \\ \alpha-\varkappa_{2}, & \alpha>1\end{cases}
$$


Proof Given two numbers $\varkappa_{1}, \varkappa_{2}, 0<\varkappa_{1}<\varkappa_{2}<1$ we define a nondecreasing function $\psi:[0,1 / 2] \rightarrow \mathbb{R}$ recursively. Let $\psi(1 / 2)=2^{-\varkappa_{1}}, t_{1}=\frac{1}{2}$. Set

$$
t_{n}^{\prime}=\left(t_{n}\right)^{\frac{1-\varkappa_{1}}{1-\varkappa_{2}}}, \quad t_{n+1}=\left(t_{n}^{\prime}\right)^{\frac{\varkappa_{2}}{\varkappa_{1}}}, \quad n \in \mathbb{N}
$$

One can write these sequences explicitly: $t_{n}=2^{-\beta^{n-1}}$, where $\beta=\frac{1-\varkappa_{1}}{1-\varkappa_{2}} \frac{\varkappa_{2}}{\varkappa_{1}}$, and $t_{n}^{\prime}=2^{-\frac{1-\varkappa_{1}}{1-\varkappa_{2}} \beta^{n-1}}, n \in \mathbb{N}$. It is clear that $t_{n+1}<t_{n}^{\prime}<t_{n}, n \in \mathbb{N}$, and $t_{n+1}=o\left(t_{n}^{\prime}\right)$, $t_{n}^{\prime}=o\left(t_{n}\right)(n \rightarrow \infty)$.

We define the function

$$
\psi(t)= \begin{cases}\left(t_{n}^{\prime}\right)^{\varkappa_{2}-1} t, & t_{n}^{\prime} \leq t \leq t_{n} \\ \left(t_{n}^{\prime}\right)^{\varkappa_{2},}, & t_{n+1} \leq t \leq t_{n}^{\prime}\end{cases}
$$

It follows from the definition of the sequences $\left(t_{n}\right)$ and $\left(t_{n}^{\prime}\right)$ that $\psi$ is a nondecreasing and continuous function on $\left[0, \frac{1}{2}\right]$. Moreover, $t^{\varkappa_{2}} \leq \psi(t) \leq t^{\varkappa_{1}}, t \in\left[0, \frac{1}{2}\right]$, and $\psi\left(t_{n}\right)=t_{n}^{\varkappa_{1}}, \psi\left(t_{n}^{\prime}\right)=\left(t_{n}^{\prime}\right)^{\varkappa_{2}}, n \in \mathbb{N}$.

It follows directly from the definition of $\psi$ that $\psi(t) / t$ is nonincreasing. By Dzyadyk and Shevchuk [7, Chap. 3] it implies semiadditivity of $\psi$, thus $\psi$ is a modulus of continuity.

Define

$$
f_{\alpha}(z)=\int_{0}^{1 / 2} \frac{d \psi(t)}{\left(1-z e^{-i t}\right)^{\alpha}}
$$

We then have

$$
f_{\alpha}(r):=\sum_{n=1}^{\infty} \int_{t_{n}^{\prime}}^{t_{n}} \frac{\left(t_{n}^{\prime}\right)^{\varkappa_{2}-1} d t}{\left(1-r e^{-i t}\right)^{\alpha}}, \quad r \in[0,1) .
$$

Since $\psi$ is a modulus of continuity, $\omega(\delta, \psi)=\psi(\delta)=O\left(\delta^{\varkappa_{1}}\right)$, and, by construction we have $\sup \left\{\gamma: \psi \in \Lambda_{\gamma}\right\}=\varkappa_{1}$. Therefore, by Theorem $\mathrm{C}$, we have that $p\left[f_{\alpha}\right] \leq$ $\alpha-\varkappa_{1}$. In fact $\left|f_{\alpha}(z)\right|=O\left((1-|z|)^{\varkappa_{1}-\alpha}\right)(|z| \rightarrow 1-)$.

Let $\alpha>1$. It follows from the construction of $\psi$ that $\liminf _{\delta \rightarrow 0+} \frac{\omega(\delta, \psi)}{\delta^{\varkappa 2}}=1$. Hence, by Theorem 3 , it follows that $\lambda\left[f_{\alpha}\right] \leq \alpha-\varkappa_{2}$. We prove that the converse inequality is true as well.

Denote

$$
I_{n}(r)=\left(t_{n}^{\prime}\right)^{\varkappa_{2}-1} \int_{t_{n}^{\prime}}^{t_{n}} \frac{\cos (\alpha \tau(t)) d t}{\left|1-r e^{-i t}\right|^{\alpha}},
$$

where

$$
\tau=\tau(t)=\arg \frac{1}{1-r e^{-i t}}, \quad \tan \tau=\frac{r \sin t}{1-r \cos t}
$$


We are going to calculate asymptotics for $I_{n}$ for an appropriate choice of $r$. Let $A_{n}=\log \frac{1}{t_{n}^{\prime}} \rightarrow+\infty(n \rightarrow+\infty)$. If $1-r \leq t_{n}^{\prime} / A_{n}$, then uniformly in $r$ and $t \in\left[t_{n}^{\prime}, t_{n}\right]$,

$$
1-r e^{-i t}=1-r+r\left(1-e^{-i t}\right) \sim i t, \quad n \rightarrow \infty .
$$

Remark 7 In what follows all asymptotic relations as $n \rightarrow \infty$ that depend on parameters $r, t$, etc. hold uniformly in these parameters at their specified ranges. For simplicity, this fact will no longer be emphasized.

We have $\tau(t) \sim \frac{\pi}{2}, \cos \alpha \tau(t) \sim \cos \frac{\alpha \pi}{2}$ as $t \in\left[t_{n}^{\prime}, t_{n}\right], n \rightarrow+\infty$. Therefore,

$$
I_{n}(r) \sim \frac{\cos \frac{\alpha \pi}{2}}{\left(t_{n}^{\prime}\right)^{1-\varkappa 2}} \int_{t_{n}^{\prime}}^{t_{n}} \frac{d t}{t^{\alpha}}=\frac{C_{0}(\alpha)+o(1)}{\left(t_{n}^{\prime}\right)^{\alpha-\varkappa_{2}}}, \quad n \rightarrow+\infty, 1-r \leq t_{n}^{\prime} / A_{n} .
$$

where $C_{0}(\alpha)=\frac{\cos \frac{\alpha \pi}{2}}{\alpha-1}$.

If $1-r \geq A_{n} t_{n}, t \in\left[t_{n}^{\prime}, t_{n}\right]$, then $\cos \alpha \tau(t) \sim 1$, and $1-r e^{-i t} \sim 1-r(n \rightarrow \infty)$, so we get

$$
I_{n}(r) \sim \frac{\left(t_{n}^{\prime}\right)^{\varkappa_{2}-1} t_{n}}{(1-r)^{\alpha}}=\frac{\left(t_{n}\right)^{\varkappa_{1}}}{(1-r)^{\alpha}}, \quad n \rightarrow+\infty .
$$

Finally, let $1-r \in\left[t_{n}^{\prime} A_{n}, t_{n} / A_{n}\right]$. We split $I_{n}$ into three integrals in this case, namely,

$$
I_{n}=\left(\int_{t_{n}^{\prime}}^{(1-r) / A_{n}}+\int_{(1-r) / A_{n}}^{(1-r) A_{n}}+\int_{(1-r) A_{n}}^{t_{n}}\right) \frac{\left(t_{n}^{\prime}\right)^{\varkappa_{2}-1} \cos (\alpha \tau(t)) d t}{\left|1-r e^{-i t}\right|^{\alpha}}=: J_{n 1}+J_{n 2}+J_{n 3} .
$$

We begin with $J_{n 2}$. Note that, $\tau(t)=\arctan \frac{r \sin t}{1-r \cos t} \sim \arctan \frac{t}{1-r}$ if $t \in[(1-$ $\left.r) / A_{n},(1-r) A_{n}\right](n \rightarrow \infty)$. Making the substitution $u=\frac{t}{1-r}$, we obtain

$$
\begin{aligned}
J_{n 2} & \sim\left(t_{n}^{\prime}\right)^{\varkappa_{2}-1} \int_{A_{n}^{-1}}^{A_{n}} \frac{\cos (\alpha \arctan u)(1-r) d u}{\left((1-r)^{2}+4 \sin ^{2} \frac{u(1-r)}{2}\right)^{\frac{\alpha}{2}}} \\
& \sim \frac{\left(t_{n}^{\prime}\right)^{\varkappa_{2}-1}}{(1-r)^{\alpha-1}} \int_{A_{n}^{-1}}^{A_{n}} \frac{\cos (\alpha \arctan u) d u}{\left(u^{2}+1\right)^{\frac{\alpha}{2}}} \sim \frac{C_{1}(\alpha)}{\left(t_{n}^{\prime}\right)^{1-\varkappa_{2}}(1-r)^{\alpha-1}}, n \rightarrow \infty,
\end{aligned}
$$

where $C_{1}(\alpha)=\int_{0}^{\infty} \frac{\cos (\alpha \arctan u) d u}{\left(u^{2}+1\right)^{\frac{\alpha}{2}}}$. Similar arguments give

$$
\begin{aligned}
& J_{n 1} \leq\left(t_{n}^{\prime}\right)^{\varkappa_{2}-1} \int_{0}^{1 / A_{n}} \frac{d t}{(1-r)^{\alpha-1}}=o\left(\frac{1}{\left(t_{n}^{\prime}\right)^{1-\varkappa_{2}}(1-r)^{\alpha-1}}\right) \\
& J_{n 3} \leq\left(t_{n}^{\prime}\right)^{\varkappa_{2}-1} \int_{(1-r) A_{n}}^{t_{n}} \frac{d t}{t^{\alpha}} \lesssim \frac{\left(t_{n}^{\prime}\right)^{\varkappa_{2}-1}}{\left(A_{n}(1-r)\right)^{\alpha-1}}=o\left(\frac{1}{\left(t_{n}^{\prime}\right)^{1-\varkappa_{2}}(1-r)^{\alpha-1}}\right)
\end{aligned}
$$


Combining (23)-(25), we get

$$
I_{n}(r) \sim \frac{C_{1}(\alpha)}{\left(t_{n}^{\prime}\right)^{1-\varkappa_{2}}(1-r)^{\alpha-1}}, \quad 1-r \in\left[t_{n}^{\prime} A_{n}, t_{n} / A_{n}\right], n \rightarrow \infty .
$$

We proceed to estimate the growth of $\left|f_{\alpha}(r)\right|$ from below.

Let now $1-r \in\left[t_{k+1} A_{k}, \frac{t_{k}^{\prime}}{A_{k}}\right]$. Then, using (21) for $n \leq k$, and (22), for $n \geq k+1$, we obtain for some $k_{0} \in \mathbb{N}$ (see Remark 7)

$$
\begin{aligned}
\Re f_{\alpha}(r) & =\left(\sum_{n=1}^{k_{0}}+\sum_{n=k_{0}+1}^{k}+\sum_{n=k+1}^{\infty}\right) I_{n} \\
& =O(1)+\sum_{n=k_{0}+1}^{k} \frac{C_{0}(\alpha)+o(1)}{\left(t_{n}^{\prime}\right)^{\alpha-\varkappa_{2}}}+\sum_{n=k+1}^{\infty}(1+o(1)) \frac{\left(t_{n}\right)^{\varkappa_{1}}}{(1-r)^{\alpha}} \\
& =O\left(\frac{1}{\left(t_{k}^{\prime}\right)^{\alpha-\varkappa_{2}}}\right)+(1+o(1)) \frac{\left(t_{k+1}\right)^{\varkappa_{1}}}{(1-r)^{\alpha}} \\
& =O\left(\frac{1}{\left(t_{k}^{\prime}\right)^{\alpha-\varkappa_{2}}}\right)+(1+o(1)) \frac{\left(t_{k}^{\prime}\right)^{\varkappa_{2}}}{(1-r)^{\alpha}} \sim \frac{\left(t_{k}^{\prime}\right)^{\varkappa_{2}}}{(1-r)^{\alpha}} \\
& \geq \frac{A_{k}^{\varkappa_{2}}}{(1-r)^{\alpha-\varkappa_{2}}}, \quad k \rightarrow+\infty .
\end{aligned}
$$

Further, let $1-r \in\left[A_{k} t_{k}^{\prime}, t_{k} / A_{k}\right]$. In this case, using (21) for $n<k$, (22) for $n \geq k+1$, and (26) for $n=k$, definitions of $\left(t_{n}\right)$ and $\left(t_{n}^{\prime}\right)$, we deduce $\left(k_{1} \in \mathbb{N}\right)$

$$
\begin{aligned}
& \Re f_{\alpha}(r)=\left(\sum_{n=1}^{k_{1}}+\sum_{n=k_{1}+1}^{k-1}+\sum_{n=k+1}^{\infty}\right) I_{n}+I_{k} \\
& =O(1)+\sum_{n=k_{1}+1}^{k-1} \frac{C_{0}(\alpha)+o(1)}{\left(t_{n}^{\prime}\right)^{\alpha-\varkappa_{2}}}+\sum_{n=k+1}^{\infty} \frac{(1+o(1))\left(t_{n}\right)^{\varkappa_{1}}}{(1-r)^{\alpha}} \\
& +\frac{C_{1}(\alpha)+o(1)}{\left(t_{k}^{\prime}\right)^{1-\varkappa_{2}}(1-r)^{\alpha-1}} \\
& =O\left(\frac{1}{\left(t_{k-1}^{\prime}\right)^{\alpha-\varkappa_{2}}}\right)+(1+o(1)) \frac{\left(t_{k+1}\right)^{\varkappa_{1}}}{(1-r)^{\alpha}}+\frac{C_{1}(\alpha)+o(1)}{\left(t_{k}^{\prime}\right)^{1-\varkappa_{2}}(1-r)^{\alpha-1}} \\
& =O\left(\frac{1}{\left(t_{k}\right)^{\frac{\varkappa_{1}}{\varkappa_{2}}\left(\alpha-\varkappa_{2}\right)}}\right)+(1+o(1)) \frac{\left(t_{k}^{\prime}\right)^{\varkappa_{2}}}{(1-r)^{\alpha}}+\frac{C_{1}(\alpha)+o(1)}{\left(t_{k}^{\prime}\right)^{1-\varkappa_{2}}(1-r)^{\alpha-1}} \\
& =O\left(\frac{1}{\left(t_{k}^{\prime}\right)^{\frac{\varkappa_{1}\left(1-\varkappa_{2}\right)}{\varkappa_{2}\left(1-\varkappa_{1}\right)}\left(\alpha-\varkappa_{2}\right)}}\right)+\frac{C_{1}(\alpha)+o(1)}{\left(t_{k}^{\prime}\right)^{1-\varkappa_{2}}(1-r)^{\alpha-1}}, \quad k \rightarrow+\infty .
\end{aligned}
$$


We show that the second addend in the last correlation dominates, i.e.

$$
\frac{1}{\left(t_{k}^{\prime}\right)^{\frac{\varkappa_{1}\left(1-\varkappa_{2}\right)}{\varkappa_{2}\left(1-\varkappa_{1}\right)}\left(\alpha-\varkappa_{2}\right)}}=o\left(\frac{1}{\left(t_{k}^{\prime}\right)^{1-\varkappa_{2}}(1-r)^{\alpha-1}}\right), \quad 1-r \in\left[A_{k} t_{k}^{\prime}, t_{k} / A_{k}\right], k \rightarrow \infty,
$$

or

$$
(1-r)^{\alpha-1}=o\left(\left(t_{k}^{\prime}\right)^{\left(1-\varkappa_{2}\right)\left(\frac{\varkappa_{1}\left(\alpha-\varkappa_{2}\right)}{\varkappa_{2}\left(1-\varkappa_{1}\right)}-1\right)}\right), \quad 1-r \in\left[A_{k} t_{k}^{\prime}, t_{k} / A_{k}\right], k \rightarrow \infty .
$$

Since $\alpha>1$ and $1-r \leq \frac{t_{k}}{A_{k}}=\frac{\left(t_{k}^{\prime}\right)^{\frac{1-\varkappa_{2}}{1-\varkappa_{1}}}}{A_{k}}$, it is sufficient to check if $\frac{(\alpha-1)\left(1-\varkappa_{2}\right)}{1-\varkappa_{1}}>$ $\left(1-\varkappa_{2}\right)\left(\frac{\varkappa_{1}\left(\alpha-\varkappa_{2}\right)}{\varkappa_{2}\left(1-\varkappa_{1}\right)}-1\right)$. This is equivalent to $\varkappa_{2}>\varkappa_{1}$, which holds by assumption. Hence, (28) can be rewritten in the form

$$
\begin{aligned}
\left|\Re f_{\alpha}(r)\right| & \sim \frac{\left|C_{1}(\alpha)\right|}{\left(t_{k}^{\prime}\right)^{1-\varkappa_{2}}(1-r)^{\alpha-1}} \\
& \geq \frac{\left|C_{1}(\alpha)\right|}{\left(\frac{1-r}{A_{k}}\right)^{1-\varkappa_{2}}(1-r)^{\alpha-1}} \\
& =\frac{\left|C_{1}(\alpha)\right| A_{k}^{1-\varkappa_{1}}}{(1-r)^{\alpha-\varkappa_{2}}}, \quad 1-r \in\left[A_{k} t_{k}^{\prime}, \frac{t_{k}}{A_{k}}\right], k \rightarrow+\infty .
\end{aligned}
$$

Therefore, taking into account (27) and (29), we obtain

$$
\left(\alpha-\varkappa_{2}\right) \log \frac{1}{1-r} \lesssim \log M(r, f) \lesssim\left(\alpha-\varkappa_{1}\right) \log \frac{1}{1-r}
$$

as $r \in \bigcup_{k=1}^{\infty}\left(\left[A_{k} t_{k+1}, \frac{t_{k}^{\prime}}{A_{k}}\right] \cup\left[A_{k} t_{k}^{\prime}, \frac{t_{k}}{A_{k}}\right]\right)$. Since for $r \in\left[t_{k+1}, t_{k}\right]$

$$
\log \left(\frac{A_{k}^{2}}{1-r}\right)^{\alpha-\varkappa_{2}} \leq \log \left(\frac{1}{1-r}\right)^{\alpha-\varkappa_{2}}+2\left(\alpha-\varkappa_{2}\right) \log A_{k} \lesssim \log \left(\frac{1}{1-r}\right)^{\alpha-\varkappa_{2}},
$$

(30) holds as $r \rightarrow 1-$ without exceptional sets.

This finishes the proof in the case $\alpha>1$.

Let now $0<\alpha<1$. We use the same function $f_{\alpha}$. Arguing similarly, we deduce

$$
I_{n}(r) \sim \frac{\cos \frac{\alpha \pi}{2}}{\left(t_{n}^{\prime}\right)^{1-\varkappa_{2}}} \int_{t_{n}^{\prime}}^{t_{n}} \frac{d t}{t^{\alpha}} \sim \frac{C_{2}(\alpha) t_{n}^{1-\alpha}}{\left(t_{n}^{\prime}\right)^{1-\varkappa_{2}}}, \quad n \rightarrow+\infty, 1-r \leq t_{n}^{\prime} / A_{n}
$$

where $C_{2}(\alpha)=\left|C_{0}(\alpha)\right|=\frac{\cos \frac{\alpha \pi}{2}}{1-\alpha}$. Correlation (22) remains true. For $J_{n m}, m \in$ $\{1,2,3\}$ we get

$$
J_{n 2} \sim \frac{\left(t_{n}^{\prime}\right)^{\varkappa_{2}-1}}{(1-r)^{\alpha-1}} \int_{A_{n}^{-1}}^{A_{n}} \frac{\cos (\alpha \arctan u) d u}{\left(u^{2}+1\right)^{\frac{\alpha}{2}}} \sim \frac{C_{2}(\alpha) A_{n}^{1-\alpha}(1-r)^{1-\alpha}}{\left(t_{n}^{\prime}\right)^{1-\varkappa_{2}}},
$$


while $J_{n 1}$ allows the same estimate as for $\alpha>1$,

$$
J_{n 3} \sim \cos \frac{\alpha \pi}{2}\left(t_{n}^{\prime}\right)^{\varkappa_{2}-1} \int_{(1-r) A_{n}}^{t_{n}} \frac{d t}{t^{\alpha}} \sim \frac{C_{2}(\alpha)}{\left(t_{n}^{\prime}\right)^{1-\varkappa_{2}}}\left(t_{n}^{1-\alpha}-\left(A_{n}(1-r)\right)^{1-\alpha}\right) .
$$

Therefore

$$
I_{n}=J_{n 1}+J_{n 2}+J_{n 3} \sim C_{2}(\alpha) \frac{t_{n}^{1-\alpha}}{\left(t_{n}^{\prime}\right)^{1-\varkappa 2}}, \quad 1-r \in\left[t_{n}^{\prime} A_{n}, t_{n} / A_{n}\right], n \rightarrow \infty .
$$

For $1-r \in\left[t_{k+1} A_{k}, \frac{t_{k}^{\prime}}{A_{k}}\right]$, as in the case $\alpha>1$, using (31) for $n \leq k$, and (22), for $n \geq k+1$, we get

$$
\begin{aligned}
& \Re f_{\alpha}(r)=O(1)+\sum_{n=k_{0}+1}^{k} \frac{\left(C_{2}(\alpha)+o(1)\right) t_{n}^{1-\alpha}}{\left(t_{n}^{\prime}\right)^{1-\varkappa_{2}}}+\sum_{n=k+1}^{\infty}(1+o(1)) \frac{\left(t_{n}\right)^{\varkappa_{1}}}{(1-r)^{\alpha}} \\
& =\frac{\left(C_{2}(\alpha)+o(1)\right) t_{k}^{1-\alpha}}{\left(t_{k}^{\prime}\right)^{1-\varkappa_{2}}}+(1+o(1)) \frac{\left(t_{k+1}\right)^{\varkappa_{1}}}{(1-r)^{\alpha}} \\
& =\frac{C_{2}(\alpha)+o(1)}{\left(t_{k}^{\prime}\right)^{\left(1-\varkappa_{2}\right) \frac{\alpha-\varkappa_{1}}{1-\varkappa_{1}}}}+(1+o(1)) \frac{\left(t_{k}^{\prime}\right)^{\varkappa_{2}}}{(1-r)^{\alpha}}, \quad k \rightarrow+\infty \text {. }
\end{aligned}
$$

We write $\tilde{t}_{n}:=\left(t_{n}^{\prime}\right)^{1+\frac{\left(\varkappa_{2}-\varkappa_{1}\right)(1-\alpha)}{\alpha\left(1-\varkappa_{1}\right)}}$. We have $t_{n+1}<\tilde{t}_{n}<t_{n}^{\prime}$, because $\varkappa_{1}<\alpha<1$. Consider two subcases. If $t_{k+1} A_{k} \leq 1-r \leq \tilde{t}_{k}$, then

$$
\begin{aligned}
\Re f_{\alpha}(r) & \geq(1+o(1)) \frac{\left(t_{k}^{\prime}\right)^{\varkappa_{2}}}{(1-r)^{\alpha}} \sim \frac{\left(\tilde{t}_{k}\right)^{\varkappa_{2} \frac{\alpha\left(1-\varkappa_{1}\right)}{\alpha\left(1-\varkappa_{2}\right)+\varkappa_{2}-\varkappa_{1}}}}{(1-r)^{\alpha}} \\
& =\frac{1}{(1-r)^{\alpha\left(1-\frac{\varkappa_{2}\left(1-\varkappa_{1}\right)}{\alpha\left(1-\varkappa_{2}\right)+\varkappa_{2}-\varkappa_{1}}\right)}}=\frac{1}{(1-r)^{\frac{\alpha\left(\alpha-\varkappa_{1}\right)\left(1-\varkappa_{2}\right)}{\alpha\left(1-\varkappa_{2}\right)+\varkappa_{2}-\varkappa_{1}}}}
\end{aligned}
$$

as required. If $\tilde{t}_{k} \leq 1-r \leq \frac{t_{k}^{\prime}}{A_{k}}$, then

$$
\Re f_{\alpha}(r) \geq \frac{C_{2}(\alpha)+o(1)}{\left(t_{k}^{\prime}\right)^{\left(1-\varkappa_{2}\right) \frac{\alpha-\varkappa_{1}}{1-\varkappa_{1}}}} \sim \frac{C_{2}(\alpha)}{\tilde{t}_{k}^{\frac{\alpha\left(1-\varkappa_{2}\right)\left(\alpha-\varkappa_{1}\right)}{\alpha\left(1-\varkappa_{2}\right)+\varkappa_{2}-\varkappa_{1}}}} \geq \frac{C_{2}(\alpha)}{(1-r)^{\frac{\alpha\left(1-\varkappa_{2}\right)\left(\alpha-\varkappa_{1}\right)}{\alpha\left(1-\varkappa_{2}\right)+\varkappa_{2}-\varkappa_{1}}}} .
$$

Thus, in both subcases we have the required lower estimate.

Finally, let $1-r \in\left[t_{k}^{\prime} A_{k}, \frac{t_{k}}{A_{k}}\right]$. Then using (31) for $n<k$, (22) for $n \geq k+1$, and (33) for $n=k$, and the definitions of $\left(t_{n}\right)$ and $\left(t_{n}^{\prime}\right)$, we deduce

$$
\Re f_{\alpha}(r)=O(1)+\sum_{n=k_{0}+1}^{k} \frac{\left(C_{2}(\alpha)+o(1)\right) t_{n}^{1-\alpha}}{\left(t_{n}^{\prime}\right)^{1-\varkappa_{2}}}+\sum_{n=k+1}^{\infty}(1+o(1)) \frac{\left(t_{n}\right)^{\varkappa_{1}}}{(1-r)^{\alpha}}
$$




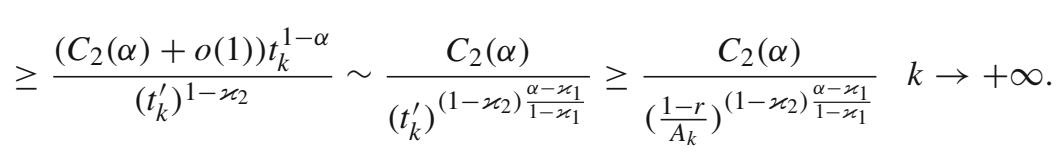

It is easy to check that $\left(1-\varkappa_{2}\right) \frac{\alpha-\varkappa_{1}}{1-\varkappa_{1}}>\frac{\alpha\left(1-\varkappa_{2}\right)\left(\alpha-\varkappa_{1}\right)}{\alpha\left(1-\varkappa_{2}\right)+\varkappa_{2}-\varkappa_{1}}$, because $\alpha<1$.

We have proved that

$$
\frac{\alpha\left(1-\varkappa_{2}\right)\left(\alpha-\varkappa_{1}\right)}{\alpha\left(1-\varkappa_{2}\right)+\varkappa_{2}-\varkappa_{1}} \log \frac{1}{1-r} \lesssim \log M(r, f) \lesssim\left(\alpha-\varkappa_{1}\right) \log \frac{1}{1-r}
$$

as $r \in \bigcup_{k=1}^{\infty}\left(\left[A_{k} t_{k+1}, \frac{t_{k}^{\prime}}{A_{k}}\right] \cup\left[A_{k} t_{k}^{\prime}, \frac{t_{k}}{A_{k}}\right]\right)(k \rightarrow+\infty)$. As in the case $\alpha>1$ we deduce that (34) holds as $r \rightarrow 1-$.

Conflict of interest Igor Chyzhykov and Galyna Beregova declare that they have no conflict of interest.

Open Access This article is distributed under the terms of the Creative Commons Attribution 4.0 International License (http://creativecommons.org/licenses/by/4.0/), which permits unrestricted use, distribution, and reproduction in any medium, provided you give appropriate credit to the original author(s) and the source, provide a link to the Creative Commons license, and indicate if changes were made.

\section{References}

1. Chyzhykov, I.: Growth and representation of analytic and harmonic functions in the unit disk. Ukr. Math. Bull. 3(1), 31-44 (2006)

2. Chyzhykov, I.: A generalization of Hardy-Littlewood's theorem. Math. Methods Physicomech. Fields 49(2), 74-79 (2006) (in Ukrainain)

3. Chyzhykov, I.: On a complete description of the class of functions without zeros analytic in a disk and having given orders. Ukr. Math. J. 59(7), 1088-1109 (2007)

4. Chyzhykov, I.: Approximation of subharmonic functions by analytic ones and asymptotic properties of meromorphic functions in a disc. Habilitation Thesis, Lviv (2008) (in Ukrainain)

5. Djrbashian, M.M.: Integral Transforms and Representations of Functions in the Complex Domain. Nauka, Moscow (1966) (in Russian)

6. Duren, P.L.: Theory of $H^{p}$ Spaces. Academic Press, London (1970)

7. Dzyadyk, V.K., Shevchuk, I.A.: Theory of Uniform Approximation of Functions by Polynomials. Walter de Gruyter, Berlin (2008)

8. Hallenbeck, D.J., MacGregor, T.H.: Radial limits and radial growth of Cauchy-Stieltjes transforms. Complex Var. 21, 219-229 (1993)

9. Hallenbeck, D.J., MacGregor, T.H.: Radial growth and exceptional sets for Cauchy-Stieltjes integrals. Proc. Edinb. Math. Soc. 37, 73-89 (1993)

10. Hardy, G.H., Littlewood, J.E.: Some properties of fractional integrals. II. Math. Z. 34, 403-439 $(1931 / 1932)$

11. Hibschweiler, R.A., MacGregor, T.H.: Fractional Cauchy Transforms. CRC, Boca Raton (2005)

12. MacGregor, T.H.: Fractional Cauchy transforms. J. Comput. Appl. Math. 105, 93-108 (1999)

13. Sheremeta, M.M.: On the asymptotic behaviour of Cauchy-Stieltjes integrals. Mat. Stud. 7(2), 175-178 (1997)

14. Stein, E.M.: Singular Integrals and Differentiability Properties of Functions. Princeton University Press, Princeton (1970)

15. Zygmund, A.: Trigonometric Series, V. 1 and 2. Cambridge University Press, Cambridge (1959)

Publisher's Note Springer Nature remains neutral with regard to jurisdictional claims in published maps and institutional affiliations. 